\title{
Passive Activity Classification Using Just WiFi Probe Response Signals
}

\author{
Fangzhan Shi \\ Department of Computer Science \\ University College London \\ London, UK \\ Email: fangzhan.shi.17@ucl.ac.uk
}

\author{
Kevin Chetty \\ Department of Security and Crime Science \\ University College London \\ London, UK \\ Email: k.chetty@ucl.ac.uk
}

\author{
Simon Julier \\ Department of Computer Science \\ University College London \\ London, UK \\ Email: s.julier@ucl.ac.uk
}

\begin{abstract}
Passive WiFi radar shows significant promise for a wide range of applications in both security and healthcare owing to its detection, tracking and recognition capabilities. However, studies examining micro-Doppler classification using passive WiFi radar have relied on manually stimulating WiFi access points to increase the bandwidths and duty-cycles of transmissions; either through file-downloads to generate high data-rate signals, or increasing the repetition frequency of the WiFi beacon signal from its default setting. In real-world scenarios, both these approaches would require user access to the WiFi network or WiFi access point through password authentication, and therefore involve a level of cooperation which cannot always be relied upon e.g. in law-enforcement applications. In this research, we investigate WiFi activity classification using just WiFi probe response signals which can be generated using a low-cost off-the-shelf secondary device (Raspberry Pi) eliminating the requirement to actually connect to the WiFi network. This removes the need to have continuous data traffic in the network or to modify the firmware configuration to manipulate the beacon signal interval, making the technology deployable in all situations. An activity recognition model based on a convolutional neural network resulted in an overall classification accuracy of $75 \%$ when trained from scratch using 300 measured WiFi proberesponse samples across 6 classes. This value is then increased to $82 \%$, with significantly less training when adopting a transfer learning approach: initial training using WiFi data traffic signals, followed by fine-tuning using probe response signals.
\end{abstract}

Keywords - Activity Recognition, Passive WiFi Doppler Radar, Probe Response, Transfer Learning

\section{INTRODUCTION}

Passive WiFi radar has the potential for a number of applications in urban areas because of its ability to detect, track and, more recently classify specific activities of personnel. For e-healthcare and ambient assisted living, the technology could be used to monitor both the frequency of movements and type of activities to infer the health status of an individual [1]. Additionally, in security and safety-critical scenarios, it could be used to detect adversaries through-walls [2], or provide indicators of signs-of-life [3]. Furthermore, unlike optical systems, WiFi radar does not generate images which could be used to identify people, and therefore maintains user privacy.

$802.11 \mathrm{~b} / \mathrm{g} / \mathrm{n}$ (WiFi) operates in the $2.4 \mathrm{GHz}$ band [4] and when exploited as a transmitter of opportunity in passive radar, has been shown to be highly sensitive to small motions [5] because of the long integration times possible. For limbs and torso movements involved with everyday human tasks, the motions are of the order of a few $\mathrm{m} / \mathrm{s}$ which would have a corresponding Doppler shift frequency of around 1-12 Hz. This is well within the Doppler resolution capability of WiFi passive radar and therefore sufficient for activity recognition [6]. WiSee [7] is an implementation of a Doppler sensing system that uses WiFi-like signals for identification of human gestures in an in-home environment with high accuracy. However, the system employs a bespoke WiFi-like narrowband $2.4 \mathrm{GHz}$ transmission signal which does not fully meet the IEEE $802.11 \mathrm{~b} / \mathrm{g} / \mathrm{n}$ standards. The WiSee receiver then analyses the received signal following a subtraction technique to obtain the frequency shift. Though WiSee is able to recognise various types of body gestures, its approach prohibits the use of commodity WiFi access points (APs) which typically employs wideband signals during the transmission of data. A number of other research studies have however proposed WiFi based passive systems that do work with commodity APs. Tan et al [8], for example have developed a system that employs highthroughput data processing techniques that enables real-time output. Two channels are used in this system: the reference channel employs a narrow-beam antenna to measure the transmissions from a WiFi AP directly, and the surveillance channel which typically makes use of a wider beam antenna for greater coverage, to measure the Doppler shifted signal from moving objects. This system design has been demonstrated experimentally using commercial wireless access points for both bistatic [9], [10] and multistatic receiver architectures [11], [12]. All of these studies assume continuous high data-rate WiFi transmissions and simulates this condition experimentally by ensuring a file is continuously being downloaded from the WiFi network via the AP. However, in real-world scenarios such as those involving the emergency services (law enforcement, counter-terrorism, search $\&$ rescue etc) this assumption is rather unrealistic and cannot be relied on as there are many instances when there are no users on the WiFi network. When the WiFi AP is idle, it continually transmits a low-bandwidth beacon signal to broadcast its presence. Some recent studies such as [6] and [13] have focused on using passive radar to exploit the WiFi beacon signal but these studies have all increased the repetition rate of the WiFi beacon bursts from every $100 \mathrm{~ms}$ (the default setting) to $20 \mathrm{~ms}$ (the maximum allowable periodicity on most WiFi APs). These adjustments to make the system usable would again require a level of cooperation with the WiFi AP (in this case password authentication) and therefore cannot be relied on for many real-world deployments.

To address the above issue, this paper takes advantage of WiFi probe response transmissions. The probe request-response protocol in the WiFi standard allows the exchange of information between the WiFi AP and client devices such as link speed, transmission power and compatibility. More importantly, we take advantage that the data in the probe protocol is not subject to any type of encryption, allowing us to repeatedly send probe requests in order to stimulate continuous wideband signal transmissions from the AP, giving us the freedom to exploit any WiFi transmitter without joining the network, or making any modifications to the AP. The probe signal has been used for cooperative outdoor localisation [14] but has not been used in any Doppler-based passive sensing system.

Doppler effect based activity recognition using machine learning 
approaches have been the subject of significant interest in the research community [15], [16], [17]. In [17] Bjorklund et al propose a feature extraction method derived from principal component analysis to select the strongest patterns from temporal Doppler records. An activity recognition classification accuracy of approximately $70 \%$ for 5-classes was achieved (walking, jogging, running, creeping and crawling). However, the high operating frequency of their commercial active radar $(77 \mathrm{GHz})$ meant that the system could only operate over a limited range. For Doppler-based activity recognition using passive WiFi radar, Chen et al [6] successfully classified a range of everyday human activities using a support vector machine (SVM) and the sparse representation classifier (SRC). In this work, two surveillance channels were employed to provide multiple aspect data for subjects under test. Moreover, automatic segmentation methods for extracting the start and end points of the micro-Doppler signature are proposed. Finally, the authors in [18] treat their Doppler radar spectrograms as static images, allowing the application of machine vision techniques. Using this approach, the authors successfully validate their premise that it is possible to transfer pre-trained convolutional neural networks for image recognition (such as GoogleNet and AlexNet) to neural networks generated from RF micro-Doppler data.

As described above, this paper attempts to examine the possibility of activity recognition using Doppler measurements from probe response transmissions to enable the passive exploitation of any $\mathrm{WiFi}$ AP. However, a key challenge is that the duty cycle of WiFi probe transmissions is lower than conventional $\mathrm{WiFi}$ data transmissions (albeit higher than WiFi beacon transmissions) which results in a reduction of classification accuracy. We therefore also apply a transfer learning strategy to fine-tune our micro-Doppler convolution neural network and improve the classification accuracy. A 500-sample micro-Doppler dataset relating to 6 classes of motions was first collected using WiFi data transmission signals. A convolution neural network is trained from scratch with this dataset. Next, the network is trained with a probe response signal dataset which was made up of 300 samples over the same 6 classes. The integration of transfer learning gave rise to a $7 \%$ increase in the classification, but more notably led to a significant reduction in training times This result has important implications for real-world deployments where there would realistically be hundreds, or even thousands, of classes of activity to be recognised.

\section{WiFi Signal GENERATION}

As explained above, many WiFi Doppler radar systems exploit high data-rate WiFi signals as opportunistic illuminators to make use of the large bandwidths and long duty cycles. However, when the network is idle with no users, the WiFi AP must be stimulated by some means (usually through file downloads) to transmit waveforms suitable for radar sensing. This approach requires the radar operator to have user access to the network, making the system unusable when the AP belongs to a third-party, or an adversary. The WiFi beacon signal has been examined as a viable alternative [6] and [13] as it is constantly transmitted by WiFi APs, although it has a much lower bandwidth. Milani et al [19] concluded that the default beacon signal interval time of $100 \mathrm{~ms}$ in commodity WiFi APs is not sufficient for WiFi sensing as the Doppler record is illegible and corrupted by noise. Our own examination of the WiFi beacon suggests the interval between bursts must be reduced to around $40 \mathrm{~ms}$ which is agreement with [19]. However, this again requires user access to the WiFi AP.

Similar to the beacon signal, the $\mathrm{WiFi}$ probe request and probe response signals are not encrypted. However, they differ in that they possess wider bandwidths and their transmissions involve longer duty cycles, and therefore offer a viable alternative opportunistic signal for use in passive WiFi radar that does not require any type of user authentication. To examine the utility of the probe signal for activity recognition we employ a Raspberry PI [20] equipped with a Kali Linux distribution to identify all WiFi APs in the immediate vicinity and attempt to handshake with any that the radar operator selects. The
Raspberry PI is then able to stimulate the WiFi AP to continuously emit probe response signals by constantly transmitting probe requests (according to the 802.11 probe protocol illustrated in Fig. 1) [4]. In our tests, the Raspberry PI was configured to transmit at its maximum frame rate limit of 75 probe requests per second with a $13 \mathrm{~ms}$ interval, though higher rates are achievable in alternative devices to generate more frequent probe response bursts.

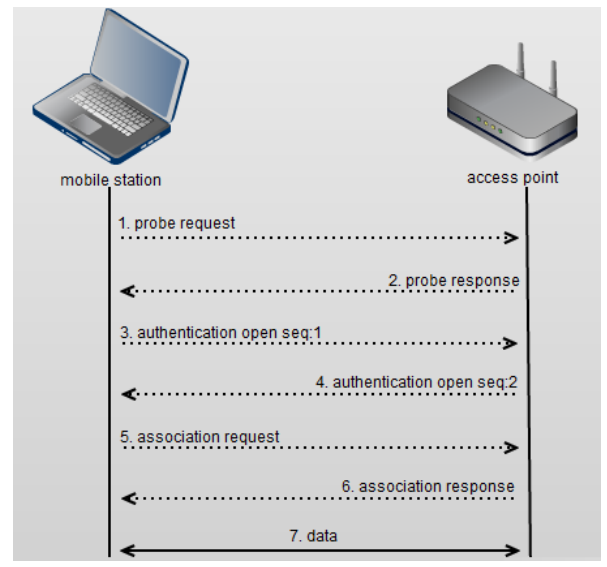

Fig. 1: WiFi Handshake Process

To assess the WiFi probe signal for passive activity classification, we undertake a campaign of data measurements. The experimental geometry is shown in Fig. 2 and a picture of the experimental apparatus are shown in Fig. 3. This setup is similar to the test environment used in [10]. It consists of a transmitter (a commodity WiFi AP) and two USRP N210 SDRs acting as reference and surveillance receivers. The reference channel employs a narrow-beam antenna directed toward the $\mathrm{WiFi} \mathrm{AP}$ to monitor its transmissions whilst minimising interference from other APs in close proximity, as well as unwanted target reflections. The surveillance channel uses a wider beamwidth antenna aimed towards a surveillance cell where test subjects undertake a range of everyday activities to generate $\mathrm{WiFi}$ micro-Doppler signatures for analysis.

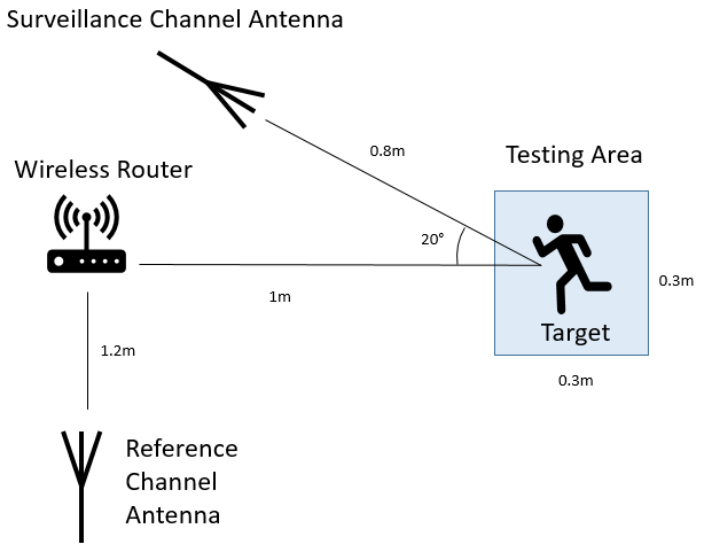

Fig. 2: Geometry Setup 


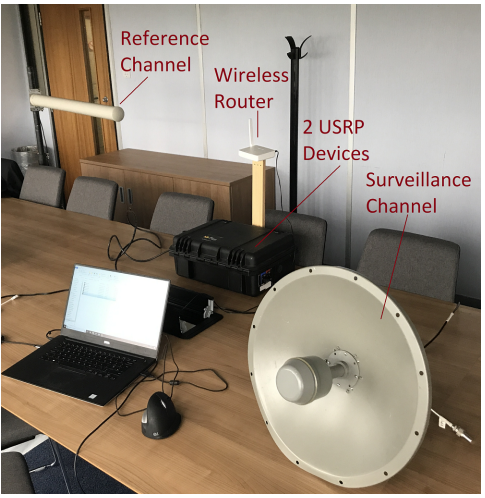

Fig. 3: Image of experimental apparatus

\section{Signal Processing and Data Collection}

To obtain the frequency shift and delay for any time-point as a subject is undertaking an activity, it is necessary to apply the discrete Cross Ambiguity Function (CAF) shown in equation (1) and described in [8]. This involves cross-correlating the data measured in the surveillance channel with Doppler shifted and delayed copies of the reference signal to identify correlations peaks that correspond to micro-motions on the body.

$$
C A F\left(\tau_{d}, f_{d}\right)=\sum_{n=0}^{N} r[n] s^{*}\left[n+\tau_{d}\right] e^{-j 2 \pi f_{d} \frac{n}{N}}
$$

In the above equation, $N$ is the total number of samples in a window, $r[\cdot]$ and $s[\cdot]$ are the discrete samples from the reference and surveillance channels respectively which is presented using complex numbers, and where $*$ is the conjugate operation. $\tau_{d}$ is the time delay which can be converted to the range, and $f_{d}$ is the Doppler frequency shift. A more detailed description of CAF processing is given in [2].

Figure 4 shows the range-Doppler surface output from the CAF processing for an example experiment. The $x$ axis is the bistatic range, and the $y$ axis is the measured Doppler frequency shift. Analysis of this result reveals a correlation peak at approximately $6 \mathrm{~Hz}$ which corresponds to a target motion of approximately $0.75 \mathrm{~m} \mathrm{~s}^{-1}$, suitable to assess micro-Doppler frequency characteristics. This peak also appears in the first target range bin but the associated range resolution of this measurement (approximately $35 \mathrm{~m}$ ) is not sufficient to generate useful localisation data. To generate micro-Doppler temporal records of human activities suitable for activity classification, the Doppler profile from the first range-bin for sequential CAF outputs are concatenated in a similar fashion to [10].

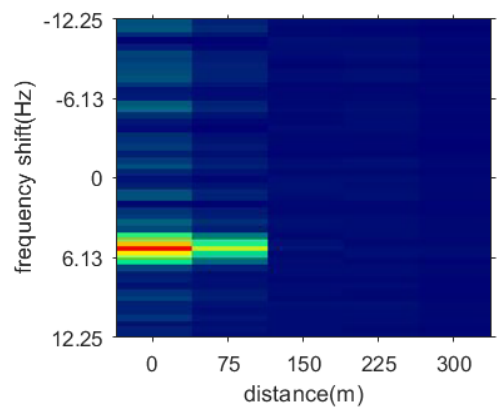

Fig. 4: Example CAF output

The experimental work involved collecting two categories of data; the first focused on measuring activity using WiFi data transmissions, and the second was concerned with WiFi probe response transmissions. For the former, a client and a server are set up to transfer data and generate a continuous high data-rate (wide bandwidth) OFDM modulated transmissions. A subject would then perform several types of motions in a test area to generate WiFi microDoppler signatures for analysis. In total 500 samples were collected across 6 classes, which are shown in Fig. 5. Each of the 6 classes had approximately 83 samples and were chosen to reflect a range of motions; They all had distinguishing upper body movements in order to generate characteristic micro-Doppler signatures. Example Doppler spectrograms from the dataset are illustrated in Fig. 6. In Fig. 6 a zero-Doppler line is present for all plots and arise from reflections of stationary objects such as walls, furniture and other everyday objects. The second $\mathrm{WiFi}$ probe response dataset is gathered using the same set of 6 classes, but with 300 samples, 50 samples for each class. Some examples are shown in Fig. 7. Comparing these Doppler spectrograms with those from Fig. 6, we can see a similar frequency response pattern for the different classes. However, the micro-Doppler traces are noisier because of the bursty nature (lower duty cycles) of $\mathrm{WiFi}$ probe-response transmissions relative to $\mathrm{WiFi}$ data transmission signals.

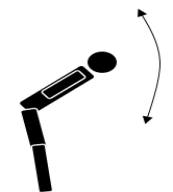

(a) Class 1

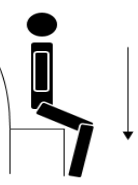

(d) Class 4

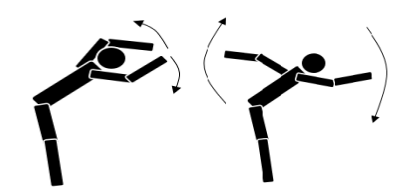

(b) Class 2

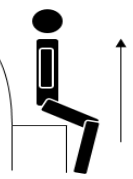

(e) Class 5 (c) Class 3

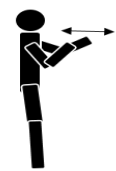

(f) Class 6
Fig. 5: Illustration of the 6 classes. Class 1: take a bow. Class 2: breast stroke. Class 3: front crawl stroke. Class 4: sit down. Class 5: stand up. Class 6: double punch.
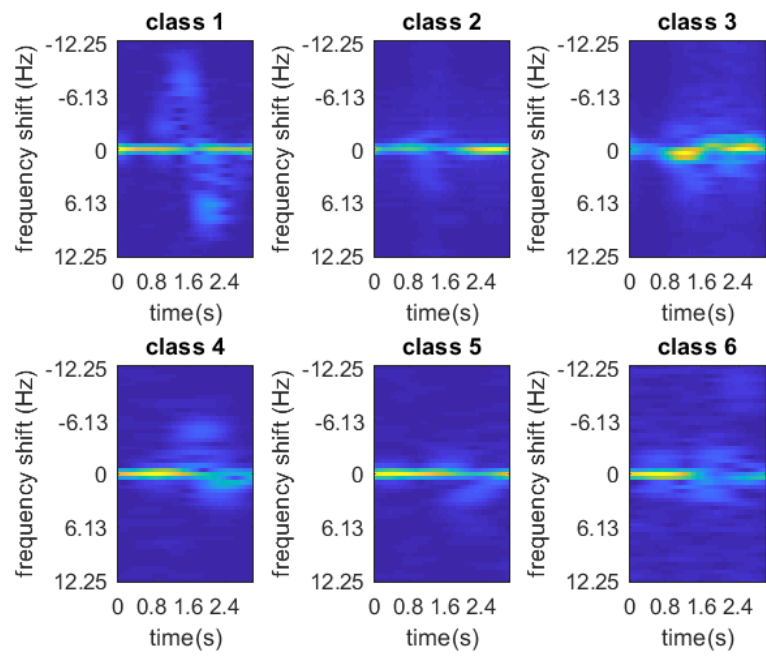

Fig. 6: Micro-Doppler measured from WiFi data transmissions 

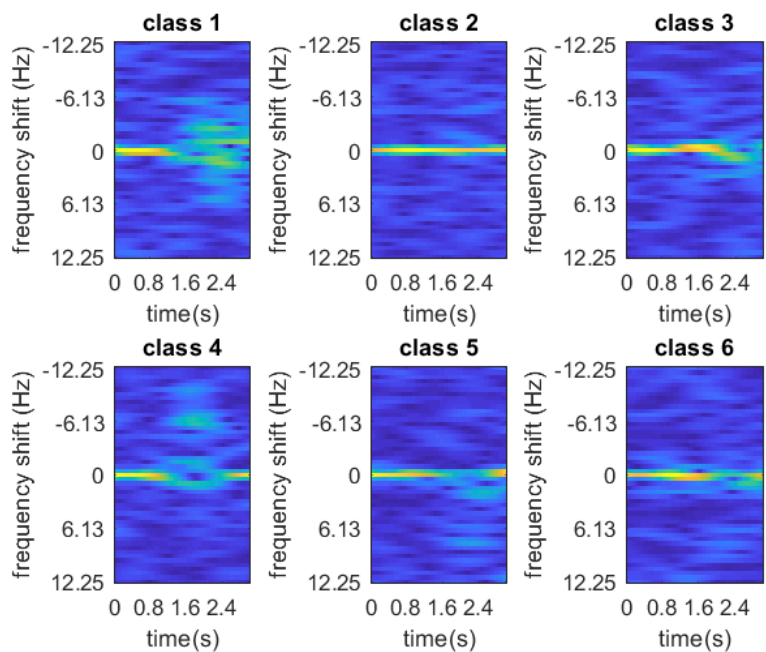

Fig. 7: Micro-Doppler from probe response transmissions

Like with other machine learning tasks, data normalisation is a critical step. For WiFi based micro-Doppler activity recognition, the power of the signal received by our passive WiFi software-defined radio (SDR) hardware may vary as the target moves around, and as the bistatic geometry changes. Our methodology for normalisation is shown below and is based on the assumption that the power remains constant over the duration of all classes of motion. For each column in the raw Doppler record, every value is first squared. Then, the values within each column are divided by the sum of the column which sets the total power for each column to unity. To suppress the noise, a two-dimensional $3 \times 3$ median filter is used to denoise the Doppler spectrogram.
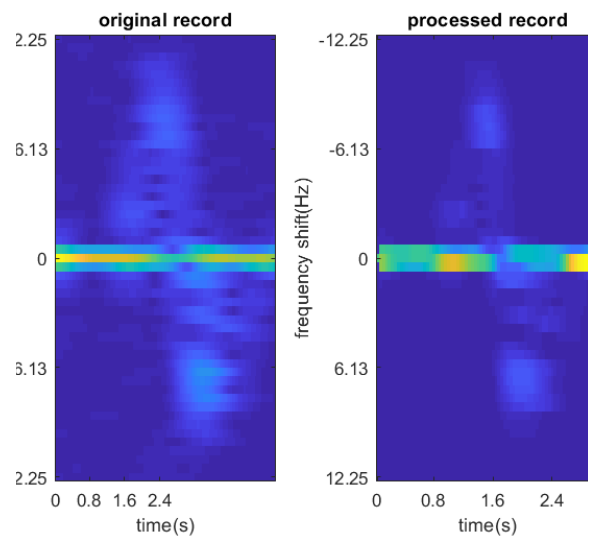

Fig. 8: A normalised and denoised Doppler record

\section{ACTIVITy ClassifiCATION}

Activity classification is defined as a classification task in which a model is able to identify a specific motion or action based on the micro-Doppler signature presented in the recorded spectrogram. As the Doppler record is stored as an image, we can use image classification techniques for activity recognition. A convolutional neural network (CNN) is a specifically designed multi-layer neural network widely used in image analysis. In this work, we propose to use a simplified version of the well-known AlexNet [21] convolution neural network. A simplified version is necessary because Alex-net is designed to process $256 \times 256$ RGB images but our Doppler records are $51 \times 75$ signal channel images. In addition, the features in the WiFi Doppler spectrograms are drawn from the frequency domain rather than a spatial domain. The proposed network is as follows: the input layer, convolution layer, ReLU layer, pooling layer, convolution layer, ReLU layer, pooling layer, fully connected layer, softmax layer and classification layer. The structure and the detailed parameters are presented in Fig. 9. Note that the ReLU layer is built into the convolution layer and therefore not shown.

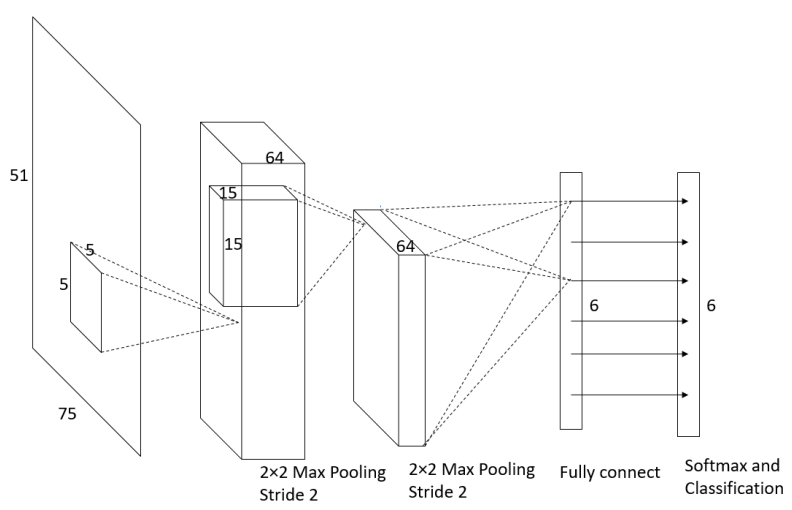

Fig. 9: The proposed $\mathrm{CNN}$ for $\mathrm{WiFi}$ activity classification

The CNN is trained and implemented in Matlab using the Neural Network Toolbox.

To test and verify the accuracy of the network, it is first trained on the WiFi data transmission measurements. $80 \%$ of the samples in the dataset are used for training and $20 \%$ are used for testing. The rate of progress in training is plotted in Fig. 10. In this training, measured samples are split into batches of 128 and the batch accuracy is defined as $\frac{\text { Number of samples correctly classified in the batch }}{\text { Number of samples in the batch }}$. In testing, the model accuracy is defined using a similar approach: $\frac{\text { Number of samples correctly classified in the test set }}{\text { Number of samples in the test set }}$. It can be seen that the batch accuracy converges to $90 \%$ and model accuracy to around $89 \%$ in the test set, suggesting no over-fitting is present. The confusion matrix is shown in Fig. 11 with true positive values that indicate the proposed network is suitable for micro-Doppler activity classification.

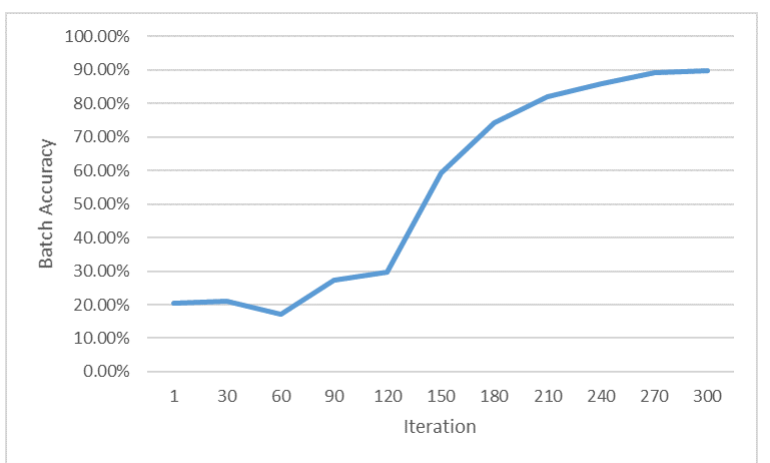

Fig. 10: The rate of progress in training the network using WiFi data signal dataset 


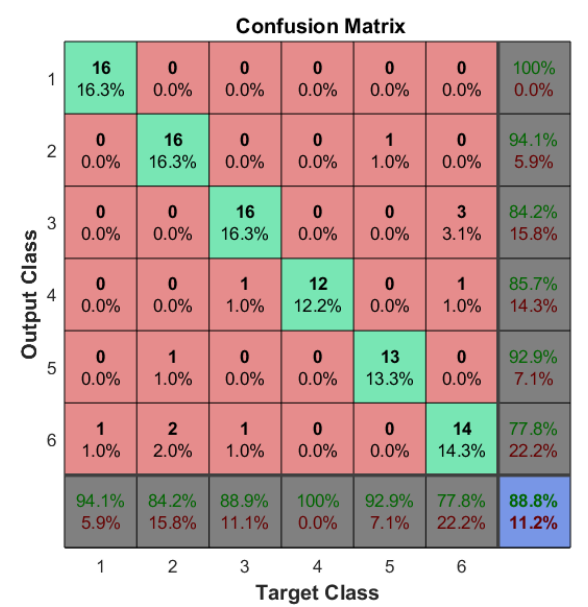

Fig. 11: Confusion matrix for WiFi data signal dataset

The second step is to re-train the $\mathrm{CNN}$ with the WiFi probe response signal dataset using transfer learning. Using a similar method, $80 \%$ samples in this dataset were used for training and $20 \%$ for testing. To compare the transfer learning approach with training from scratch, a CNN with an identical structure was created and trained only with the WiFi probe response signal dataset. To ensure a fair comparison, both of the models were trained using 300 iterations. The training progress rate is shown in Fig. 12. It is clear that the accuracy of the CNN using transfer learning surges after just the first few iterations while the accuracy of the $\mathrm{CNN}$ trained from scratch remains relatively low. The accuracy of the model trained from scratch rises at around 110 iterations but at that stage, the transfer learning model is already approaching the maximum accuracy. Towards the end (around 300 iterations) both converge: For the WiFi probe response signal dataset, the accuracy of transfer learning is $81.7 \%$ while the accuracy of the model trained from scratch is $75.0 \%$. The confusion matrices are shown in Fig. 13. This result highlights the benefit of adopting a transfer learning approach to ensure feasible training times when for example developing a commercial activity recognition system that would require identification of hundreds, or even thousands of classes. It is, however, worth noting that the final batch accuracy in the transfer learning progress approaches $98 \%$ but the actual performance on the test set is $81.7 \%$ indicating the presence of over-fitting.

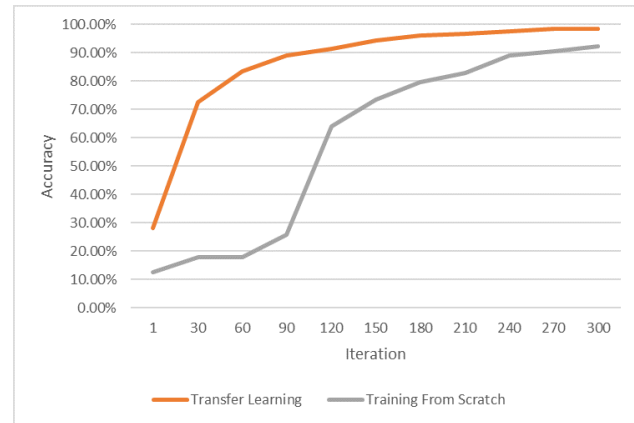

Fig. 12: Comparison of transfer learning and training from scratch

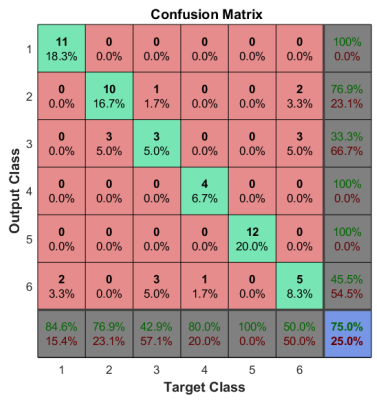

(a) Training from scratch

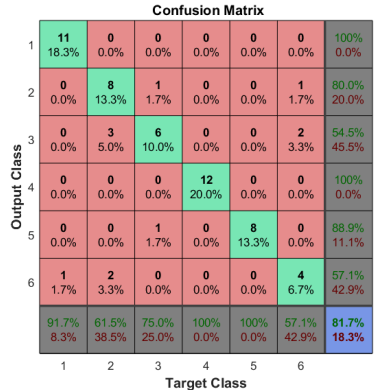

(b) Transfer learning
Fig. 13: Confusion matrix of the $\mathrm{CNN}$ trained with WiFi probe response signal dataset

\section{Summary AND Discussion}

In real-world applications of WiFi passive radar, a system would ideally exploit the 802.11 OFDM signals due to their high-bandwidths and long duty cycles. However, given that these transmissions are not guaranteed, and the fact that the everpresent WiFi beacon signal with $100 \mathrm{~ms}$ burst interval is not suitable for micro-Doppler analysis, the WiFi probe response signal provides the most reliable and viable option for micro-Doppler based recognition and therefore should feature as a core part of a sensing toolkit. In this case, taking advantage of the transfer learning is a practical approach to train the network models employed in a fast and efficient manner, especially as a commercial system would be expected to identify a much broader range of motion types and activities. Other considerations for realworld deployment of such a technology include training the networks using data gathered in different environmental conditions and over a larger range of bistatic geometries. There is also a requirement to deal with unsegmented Doppler records.

The transfer learning approach which we have adopted in this work is able to speed up the training time in our 6 class classification by a factor of approximately 2 . By determining the image of the spectrogram which most frequently stimulates a neuron in the CNN to fire, we are able to identify the features learned by the neural network trained using the WiFi data transmission signals. Fig. 14 illustrates features learned by the second convolution layer of the $\mathrm{CNN}$, and highlights the abstract nature of the feature set; it consists of slopes, horizontal lines, dashed lines, and even noise-like patterns. The isolation of these abstract features require significant computational time and resource but are inherited through the process of transfer learning which gives rise to more efficient, and therefore faster training time .compared to training from scratch. Both approaches do however lead to similar accuracy and seem to be limited by (a) the capability of the recognition model and (b) the quality of the dataset.

One of the main limitations of our approach is that the probe response signals, which act as the opportunistic illuminator are stimulated by continually sending probe requests signal to the $\mathrm{WiFi}$ AP. These act as an unwanted source of noise in the radar receivers and can mask target responses. Currently, we minimise interference effects manually by choosing a probe request transmission power just above the threshold to stimulate the probe response, though a real-world system would ideally set this parameter automatically. Moreover, classification performance may degrade as the distance between the stimulation device (in our case a Rasberry Pi) and WiFi AP increases. 


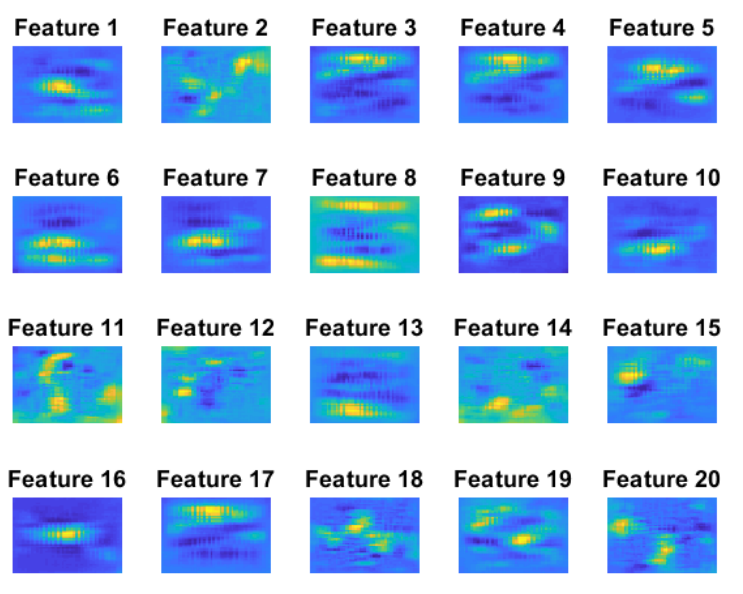

Fig. 14: Features learned by second convolution layer in CNN trained using WiFi Data Signal Dataset

\section{CONCLUSiOn AND Future Work}

This paper presents a WiFi-based passive activity recognition system that just uses the WiFi probe signal which can work with any wireless AP, even if it is protected by a password or other authentication methods. By repetitively sending a WiFi probe request signal, the wireless AP is stimulated to continually transmit a WiFi probe signal which can be exploited as a reliable illuminator of opportunity. Two sets of experimentally measured activity data are gathered; one using WiFi transmissions containing data traffic, and other using just WiFi probe response signals. CNN models that were either trained from scratch or trained using a transfer learning were assessed and compared and it was found that although they achieved similar classification accuracy across 6 classes of activity $(75 \%$ and $82 \%$ respectively). Additionally, transfer learning reduced training times 2-fold, which would be highly desirable when training networks that are required to classify across significantly more classes. Finally, the in our current system the recognition tasks are implemented offline so to achieve our future objective of moving towards a realworld commercial system, we aim to investigate edge-computing methodologies to will facilitate real-time classification on-board our SDR prototype.

\section{REFERENCES}

[1] B. Tan, Q. Chen, K. Chetty, K. Woodbridge, W. Li, and R. Piechocki, "Exploiting WiFi Channel State Information for Residential Healthcare Informatics," IEEE Communications Magazine, vol. 56, no. 5, pp. 130-137, may 2018. [Online]. Available: https://ieeexplore.ieee.org/document/8360863/

[2] K. Chetty, G. E. Smith, and K. Woodbridge, "Through-the-Wall Sensing of Personnel Using Passive Bistatic WiFi Radar at Standoff Distances," IEEE Transactions on Geoscience and Remote Sensing, vol. 50, no. 4, pp. 1218-1226, apr 2012. [Online]. Available: http://ieeexplore.ieee.org/document/6020778/

[3] Q. Chen, K. Chetty, K. Woodbridge, and B. Tan, "Signs of life detection using wireless passive radar," in 2016 IEEE Radar Conference (RadarConf). IEEE, may 2016, pp. 1-5. [Online]. Available: http://ieeexplore.ieee.org/document/7485313/

[4] IEEE Computer Society. LAN/MAN Standards Committee., Institute of Electrical and Electronics Engineers., and IEEE-SA Standards Board., IEEE standard for information technology : telecommunications and information exchange between systems : local and metropolitan area networks-specific requirements. Part 11, Wireless LAN medium access control (MAC) and physical layer (PHY) specifications. Institute of Electrical and Electronics Engineers, 2012.
[5] B. Tan, K. Woodbridge, and K. Chetty, "Awireless passive radar system for real-time through-wall movement detection," IEEE Transactions on Aerospace and Electronic Systems, vol. 52, no. 5, pp. 2596-2603, oct 2016. [Online]. Available: http://ieeexplore.ieee.org/document/7812900/

[6] Q. Chen, B. Tan, K. Chetty, and K. Woodbridge, "Activity recognition based on micro-Doppler signature with in-home Wi-Fi," in 2016 IEEE 18th International Conference on e-Health Networking, Applications and Services, Healthcom 2016. IEEE, sep 2016, pp. 1-6. [Online]. Available: http://ieeexplore.ieee.org/document/7749457/

[7] Q. Pu, S. Gupta, S. Gollakota, and S. Patel, "Whole-Home Gesture Recognition Using Wireless Signals," Acm Mobicom, pp. 485486, 2013. [Online]. Available: https://wisee.cs.washington.edu/wisee paper.pdf

[8] B. Tan, K. Woodbridge, and K. Chetty, "A real-time high resolution passive WiFi Doppler-radar and its applications," in 2014 International Radar Conference. IEEE, oct 2014, pp. 1-6. [Online]. Available: http://ieeexplore.ieee.org/document/7060359/

[9] F. Colone, P. Falcone, C. Bongioanni, and P. Lombardo, "WiFiBased Passive Bistatic Radar: Data Processing Schemes and Experimental Results," IEEE Transactions on Aerospace and Electronic Systems, vol. 48, no. 2, pp. 1061-1079, 2012. [Online]. Available: http://ieeexplore.ieee.org/document/6178049/

[10] K. Chetty, B. Tan, and K. Woodbridge, "Data processing for real-time wireless passive radar," in 2014 IEEE Radar Conference. IEEE, may 2014, pp. 0455-0459. [Online]. Available: http://ieeexplore.ieee.org/lpdocs/epic03/wrapper.htm?arnumber=6875634

[11] S. Rzewuski, M. Wielgo, K. Kulpa, M. Malanowski, and J. Kulpa, "Multistatic passive radar based on WIFI - Results of the experiment," in 2013 International Conference on Radar. IEEE, sep 2013, pp. 230234. [Online]. Available: http://ieeexplore.ieee.org/document/6651990/

[12] M. Broetje, "Person tracking for WiFi based multistatic passive radar," undefined, 2013. [Online]. Available: $\quad$ https://www.semanticscholar.org/paper/Persontracking-for-WiFi-based-multistatic-passive-

Broetje/8296ab3acddc5337682e4a8610c13c441c18b345

[13] W. Li, B. Tan, R. J. Piechocki, and I. Craddock, "Opportunistic physical activity monitoring via passive WiFi radar," in 2016 IEEE 18th International Conference on e-Health Networking, Applications and Services (Healthcom). IEEE, sep 2016, pp. 1-6. [Online]. Available: http://ieeexplore.ieee.org/document/7749458/

[14] V. Acuna, A. Kumbhar, E. Vattapparamban, F. Rajabli, and I. Guvenc, "Localization of WiFi Devices Using Probe Requests Captured at Unmanned Aerial Vehicles," in 2017 IEEE Wireless Communications and Networking Conference (WCNC). IEEE, mar 2017, pp. 1-6. [Online]. Available: http://ieeexplore.ieee.org/document/7925654/

[15] B. Jokanovic and M. Amin, "Fall Detection Using Deep Learning in Range-Doppler Radars," IEEE Transactions on Aerospace and Electronic Systems, vol. 54, no. 1, pp. 180-189, feb 2018. [Online]. Available: http://ieeexplore.iee.org/document/8010417/

[16] Y. Shao, Y. Dai, L. Yuan, and W. Chen, "Deep Learning Methods for Personnel Recognition based on Micro-Doppler Features," in Proceedings of the 9th International Conference on Signal Processing Systems - ICSPS 2017. New York, New York, USA: ACM Press, 2017, pp. 94-98. [Online]. Available: http://dl.acm.org/citation.cfm?doid=3163080.3163095

[17] S. Bjorklund, T. Johansson, and H. Petersson, "Evaluation of a micro-Doppler classification method on mm-wave data," in 2012 IEEE Radar Conference. IEEE, may 2012, pp. 0934-0939. [Online]. Available: http://ieeexplore.ieee.org/document/6212271/

[18] M. S. Seyfioglu and S. Z. Gurbuz, "Deep Neural Network Initialization Methods for Micro-Doppler Classification With Low Training Sample Support," IEEE Geoscience and Remote Sensing Letters, vol. 14, no. 12, pp. 2462-2466, dec 2017. [Online]. Available: http://ieeexplore.ieee.org/document/8119733/

[19] I. Milani, F. Colone, C. Bongioanni, and P. Lombardo, "Impact of Beacon Interval on the performance of WiFi-based passive radar against human targets," in 2018 22nd International Microwave and Radar Conference (MIKON). IEEE, may 2018, pp. 190-193. [Online]. Available: https://ieeexplore.ieee.org/document/8405175/

[20] Raspberry Pi Foundation, "Raspberry Pi Documentation," 2018. [Online]. Available: https://www.raspberrypi.org/documentation/

[21] A. Krizhevsky, I. Sutskever, and G. E. Hinton, "ImageNet classification with deep convolutional neural networks," pp. 1097-1105, 2012. [Online]. Available: https://dl.acm.org/citation.cfm?id=2999257 